\title{
Ein Arzt im Selbstversuch
}

\section{Willy Stoll}

Prof. Dr. med., ehem. Kantonsspital Aarau

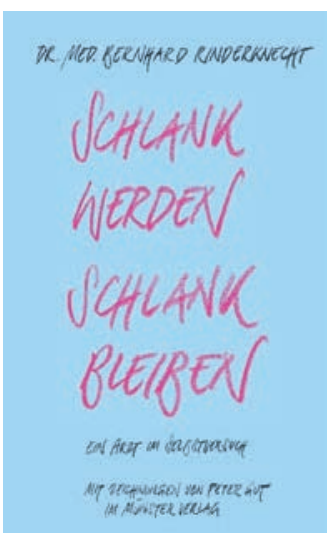

Bernhard Rinderknecht

Schlank werden - schlank bleiben

Basel: Münster Verlag; 2018.

95 Seiten.

ISBN 978-3-907146-04-0

\section{Mit freudbetonter Grundstimmung}

Der Autor des kürzlich im Münster Verlag Basel herausgekommenen kleinen Bandes Schlank werden schlank bleiben, Dr. med. Bernhard Rinderknecht, absolvierte seine Ausbildung zum Facharzt Gynäkologie und Geburtshilfe an der Frauenklinik des Kantonsspitals Aarau. Er war tüchtig, liebenswürdig, zielstrebig. Nur lag sein Körpergewicht deutlich über der Norm. Es blieb uns nicht verborgen, dass dieser Makel sein Wohlbefinden trübte.

Beruflich führte seine Erfolgskurve aufwärts, weiter stieg auch sein Gewicht. Dann - nach Jahren - trat er

\section{Essen hat einen grösseren Einfluss,} aber Bewegen ist wichtiger.

uns verändert entgegen: strahlend und schlank. Seine sportlichen Aktivitäten liessen uns klein werden. Wohl verriet sein Lächeln einen gewissen Stolz, aber da wurde keine Verbissenheit, keine eiserne Disziplin überspielt, da musste sich eine freudbetonte Grundstimmung eingestellt haben. Davon berichtet er in der vorliegenden Schrift.

In kurzgefassten, spielend leicht lesbaren Abschnitten führt er uns in seine Gedankenwelt ein. Er hat nachgedacht und nachgeforscht. Übergewicht ist kein monofaktorielles Problem und lässt sich nicht mit einer einzigen Massnahme lösen.

\section{Keine Schuldgefühle!}

Es gibt eine immense Ratgeberliteratur. Bernhard Rinderknecht warnt: Haltet euch davon fern. Sein kleiner Band kommt bescheiden daher, und er nennt die Grundlagen für Abnehmwillige: Interesse, Neugier, Geduld. Er will von Schuldgefühlen befreien. Man muss nicht MÜSSEN.

Es geht um eine Prioritätenfindung, tragend ist das «ich will». Zur Entfaltung von Neugier und zum Aufkommen von Freude braucht es zeitliche Freiräume. Wenn Wichtigeres ansteht, soll man sich mit der Last der Übergewichtigkeit nicht noch der Frustration fehlgeschlagener Versuche zum Abnehmen aussetzen. Unser Leben hat eine unnatürliche Beschleunigung erfahren, es fehlt an Geduld.

\section{Sein kleiner Band kommt bescheiden daher,} und er nennt die Grundlagen für Abnehmwillige: Interesse, Neugier, Geduld.

Eingestreut in die Textfolge sind gut verständliche, sogar amüsante Hinweise zu physiologischen und evolutionsbiologischen Zusammenhängen. Talent und Erfahrung des Dozenten werden spürbar.

\section{Wo beginnen mit dem Abnehmen?}

Mit mehr Bewegung! Mit Wandern, Walking, Joggen, Schwimmen, Fahrradfahren u.a.m. erfahren wir gleichsam neu die Schönheiten von Landschaften und Jahreszeiten. Neugier und Freude sind stärker als Disziplin. Am besten beginnt man alleine, sachte anfangen, langsam steigern. Man soll angeregt und nicht erschöpft sein. Die Ausdauerleistungsfähigkeit wird zu 
etwa 90\% nach drei Monaten erreicht. Irgendwelche elektronischen Kontrollgeräte braucht es nicht, das Gefühl ist massgebend. Die Stimmung hebt sich, der Kopf wird freier, plötzlich werden Probleme lösbar. Essen hat einen grösseren Einfluss, aber Bewegen ist WICHTIGER, insbesondere langfristig gesehen. Bewegen und Essen werden mit positiven emotionalen Erlebnissen verbunden. Zum Essen sind einige Fakten grundlegend, für das Bewegen genügen einfach verständliche Regeln.

\section{Die neuen Essgewohnheiten müssen genussreich sein}

Das Risiko, wieder zuzunehmen, verläuft parallel zur Reduktion der körperlichen Aktivität und zum Fettgehalt der Ernährung. Den neuen Essgewohnheiten (fetthaltige Komponenten sind zu reduzieren, Gemüse und Früchte zu favorisieren) soll man treu bleiben. Aber diese neuen Essgewohnheiten müssen genussreich sein. Nie soll man gedankenlos essen. Es liegt halt bei der Übergewichtigkeit an den Genen!? Gewiss hat die genetische Grunddisposition einen grossen Anteil daran, ob jemand übergewichtig wird, aber immerhin ein Drittel dieser Einflussfaktoren ist unabhängig von der Disposition und kann durch körperliche Aktivität entscheidend beeinflusst werden. Der Mensch ist mit der Übergewichtigkeit nicht einem ehernen Gesetz ausgeliefert.

\section{Die Waage ist keine rote Verkehrsampel}

Erfolgreiche Abnehmwillige (und übrigens auch Leistungssportler) wägen sich regelmässig. Man soll die Waage nicht als eine drohend auf Rot gestellte Verkehrsampel betrachten. Man kann spielerisch mit ihr umgehen. Nur schon der Unterschied zwischen Abendund Nüchterngewicht am Morgen ist interessant, und mit der Zeit macht es Spass, vorauszusagen, welche Anzeige im Display der Waage aufleuchten wird.

Im Zenit seines beruflichen Lebens als praktizierender Gynäkologe und Leiter eines grossen Labors setzte Bernhard Rinderknecht neue Prioritäten. Im Laufe einiger Jahre gelang es ihm, sein Gewicht von 133 auf $83 \mathrm{~kg}$ zu reduzieren, um sagenhafte $50 \mathrm{~kg}$ ! Mit seinem Wohlbefinden stieg seine sportliche Leistungsfähigkeit; schon zweimal hat er den Ironman mitgemacht.

\section{Man soll die Waage nicht als eine drohend auf Rot gestellte Verkehrsampel betrachten.}

Sein kleines Buch ist gediegen ausgestattet. Die von Peter Gut (NZZ, Bilanz) mit souveränem Strich gestalteten, mit feinem Witz und einer Prise Satire unterlegten Illustrationen reflektieren in schöner Weise die vom Verfasser in schlichter Sprache vorgetragenen Überlegungen. 